\title{
Preparation and Characterization of Reduced Graphene Oxide Sheets via Water-Based Exfoliation and Reduction Methods
}

\author{
Vorrada Loryuenyong, ${ }^{1,2}$ Krit Totepvimarn, ${ }^{1}$ Passakorn Eimburanapravat, ${ }^{1}$ \\ Wanchai Boonchompoo, ${ }^{1}$ and Achanai Buasri ${ }^{1,2}$ \\ ${ }^{1}$ Department of Materials Science and Engineering, Faculty of Engineering and Industrial Technology, Silpakorn University, \\ Nakhon Pathom 73000, Thailand \\ ${ }^{2}$ National Center of Excellence for Petroleum, Petrochemicals and Advanced Materials, Chulalongkorn University, \\ Bangkok 10330, Thailand \\ Correspondence should be addressed to Vorrada Loryuenyong; vorrada@gmail.com
}

Received 29 May 2013; Revised 3 August 2013; Accepted 9 August 2013

Academic Editor: Peter Majewski

Copyright (C) 2013 Vorrada Loryuenyong et al. This is an open access article distributed under the Creative Commons Attribution License, which permits unrestricted use, distribution, and reproduction in any medium, provided the original work is properly cited.

\begin{abstract}
This research studied the synthesis of graphene oxide and graphene via a low-cost manufacturing method. The process started with the chemical oxidation of commercial graphite powder into graphite oxide by modified Hummer's method, followed by the exfoliation of graphite oxide in distilled water using the ultrasound frequency from a laboratory ultrasonic bath. Finally, the oxygen functional groups on exfoliated graphite oxide or graphene oxide were eliminated by stirring in hot distilled water at $95^{\circ} \mathrm{C}$, as a replacement for highly toxic and dangerously unstable hydrazine. The results assured that stirring in hot distilled water could give the product of graphene or reduced graphene oxide. The samples were characterized by FTIR, XRD, TGA, Raman spectroscopy, SEM, and TEM methods.
\end{abstract}

\section{Introduction}

Graphene is a one-atom-thick planar layer of $\mathrm{sp}^{2}$ hybridization carbon atoms which arrange in a hexagonal structure. It has unique and remarkable mechanical, thermal, optical, and electrical properties. In addition, the two-dimensional structure causes graphene to have a zero band gap and to act as a semimetal $[1,2]$. Graphene can be synthesized by various methods such as chemical vapor deposition, mechanical exfoliation and cleavage, and annealing a single-crystal SiC under ultrahigh vacuum. These methods, however, have many disadvantages including high energy requirement, low yield, and limitation of instrument. Up to now, the chemical method has become a promising route to produce graphene sheets, although graphene derived by this method could contain a significant amount of oxygen functional groups and defects. This is because it is simple, inexpensive, and suitable for large-scale or mass production. The process involves 3 important steps: graphite oxidation, exfoliation of graphite oxide, and reduction of graphene oxide sheets.
In the first step, bulk graphite would be oxidized by chemical oxidation to become hydrophilic graphite oxide (GO). Graphite oxide is then exfoliated into monolayers or few-layered stacks of graphene oxide (XGO). This could be achieved by various thermal and mechanical methods. Sonicating or stirring in water is the most common. Finally, graphene oxide is reduced into graphene-like material. Previous works have reported the reduction by using reductants such as hydrazine. However, these chemicals might be highly toxic to human and environment $[3,4]$. This has, therefore, stimulated a number of researches in recent years to develop simple methods for the reduction of graphene oxide. Liao et al. [5], for example, have reported a new simple, nontoxic and hydrazine-free method to reduce graphene oxide by stirring in hot water under acid condition.

In this paper, we first treated graphite powders with concentrated sulfuric and potassium permanganate, using modified Hummer's method, to produce graphite oxide. The process was designed to be simple and with a low risk, so that can be done in collage's laboratory. Graphite oxide was then 
exfoliated and reduced into reduced graphene oxide (RGO) by ultrasonication in water and stirring in hot water. The obtained samples were studied by FTIR, XRD, TGA, Raman spectroscopy, SEM, and TEM methods. The objective of this work is to study the feasibility to simply synthesize reduced graphene oxide in a weak acid solution.

\section{Materials and Methods}

2.1. Materials. Graphite (fine powder extra pure) and hydrochloric acid fuming (37\%) were purchased from Merck Ltd., Germany. Sulfuric acid (98\%) Grade AR was purchased from Mallinckrodt Chemical Ltd. Potassium permanganate was purchased from Ajax Finechem Pty Ltd. Hydrogen peroxide (30-32\%) Grade AR was purchased from QReC chemical co., Ltd. All chemicals were used as received.

2.2. Synthesis of Graphite Oxide (GO). Graphite oxide was synthesized from graphite powder by modified Hummer's method [6]. 2.0 g of graphite powder was first added into $100 \mathrm{~mL}$ concentrated $\mathrm{H}_{2} \mathrm{SO}_{4}$ at room temperature. Under stirring, the mixture was cooled to $5^{\circ} \mathrm{C}$ using an ice bath, and the temperature of the mixture was kept to be below $5^{\circ} \mathrm{C}$ for $30 \mathrm{~min} . \mathrm{KMnO}_{4}(8.0 \mathrm{~g})$ was then added gradually under stirring and cooling, so that the temperature of the mixture was not allowed to reach $10^{\circ} \mathrm{C}$. $100 \mathrm{~mL}$ distilled water was added into the mixture, stirred for $1 \mathrm{hr}$., and further diluted to approximately $300 \mathrm{~mL}$ with distilled water. After that, $20 \mathrm{~mL}$ of $30 \% \mathrm{H}_{2} \mathrm{O}_{2}$ was added to the mixture to reduce the residual $\mathrm{KMnO}_{4}$. The solid was filtered, washed with $5 \% \mathrm{HCl}$ aqueous solution $(800 \mathrm{~mL})$ to remove metal ions and with distilled water until the $\mathrm{pH}$ was 6 . The resulting graphite oxide (GO) was dried at $45^{\circ} \mathrm{C}$ for $24 \mathrm{~h}$.

2.3. Synthesis of Exfoliated Graphite Oxide (XGO). Aqueous colloids of XGO were prepared by dispersing $1.0 \mathrm{~g}$ GO into $500 \mathrm{~mL}$ of distilled water by ultrasonication and centrifugation for $2 \mathrm{hr}$. to remove any unexfoliated GO. The XGO was dried at $45^{\circ} \mathrm{C}$ for $24 \mathrm{~h}$.

2.4. Synthesis of Reduced Graphene Oxide (RGO). The RGO was prepared by dispersing $400 \mathrm{mg}$ XGO into $800 \mathrm{~mL}$ of distilled water while stirring and heating at $95^{\circ} \mathrm{C}$ for 4 days.

2.5. Characterization. Thermogravimetric analysis (TGA) was performed in a Perkin Elmer (TGA7) from 50 to $800^{\circ} \mathrm{C}$ under nitrogen atmosphere (heating rate: $10^{\circ} \mathrm{C} / \mathrm{min}$ ). Fourier transform infrared spectroscopy (FTIR) was performed on a Bruker (Vertex 70). X-ray diffraction (XRD) patterns were recorded on a Rigaku (Miniflex II), employing a scanning rate of $0.033^{\circ} \mathrm{s}^{-1}$ from $5^{\circ}$ to $60^{\circ}$ with CuK $\alpha$ radiation $(k=0.154 \mathrm{~nm})$. The morphology of as-prepared samples was characterized using scanning electron microscope (SEM, Camscan: MX 200) and transmission electron microscope (TEM, JEOL: JEM-1230) operating at 80-120 kV. The absorbance of sample solutions was detected by UV-Vis Spectrophotometer (Shimadzu: UV-1800). Fourier transform raman spectrometer (FT-Raman, Perkin Elmer Spectrum

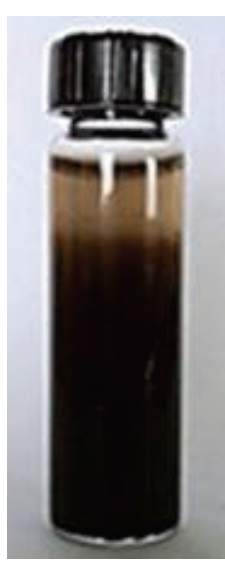

(a)

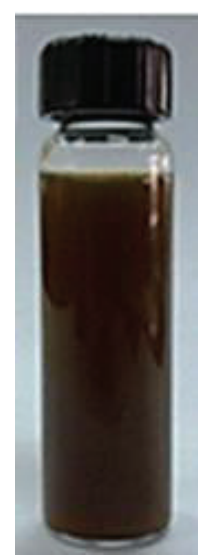

(b)

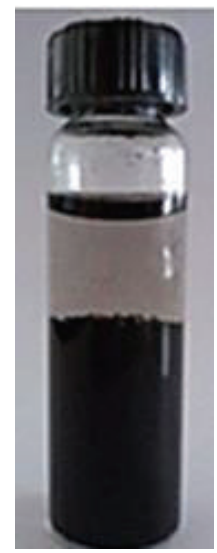

(c)
FIGURE 1: (a) Graphite oxide, (b) XGO, and (c) RGO.

GX) invariably incorporated with Nd:YAG solid state lasers operating at $1064 \mathrm{~nm}$ was used to characterize functional groups and structural information.

\section{Results and Discussion}

Figure 1 shows the obtained graphite oxide, graphene oxide, and reduced graphene oxide suspension in water. Graphite oxide prepared by a modified Hummer's method creates a brown aqueous suspension when dispersed in water as shown in Figure 1(a). The exfoliation of bulk graphite oxide in an ultrasonic bath results in a brown color and semitransparency of graphene oxide (Figure 1(b)). The negative surface charge of graphene oxide induces electrostatic repulsion among them and making it strongly hydrophilic [7]. However, when the graphene oxide was reduced in hot water, black precipitates are formed (Figure 1(c)). This is a typical hydrophobic characteristic of RGO or graphene sheets with less polar functionality on the surface of the sheets, compared to that of XGO [8].

Thermal stability of graphite, XGO and RGO was examined by TGA analysis (Figure 2). It is evident that graphite exhibits one clear step of weight loss, while XGO decomposes in three steps. The first weight loss at $50-120^{\circ} \mathrm{C}$ relates to the loss of water molecules. The second step observed from $120^{\circ} \mathrm{C}$ to $440^{\circ} \mathrm{C}$ in $\mathrm{XGO}$ is due to the loss of oxygen-containing groups, and the third step above $440^{\circ} \mathrm{C}$ relates to an unstable carbon remaining in the structure and the pyrolysis of oxygen functional groups in the main structure to yield $\mathrm{CO}$ and $\mathrm{CO}_{2}$ [9-11]. The RGO shows similar characteristic but with lower amount of weight loss, compared to that of XGO. This could be explained by a smaller amount of oxygen functional groups in the structure.

The FTIR spectra of graphite and XGO are presented in Figures 3(a) and 3(b), respectively. The graphs show the stretching of hydroxyl group at $3450 \mathrm{~cm}^{-1}$, the $\mathrm{C}=\mathrm{O}$ carbonyl stretching at $1728 \mathrm{~cm}^{-1}$, and the $\mathrm{C}-\mathrm{O}$ epoxide group stretching at 1229 and $1061 \mathrm{~cm}^{-1}$ [12]. The high intensity of the main peaks in XGO confirms the presence of a large amount 


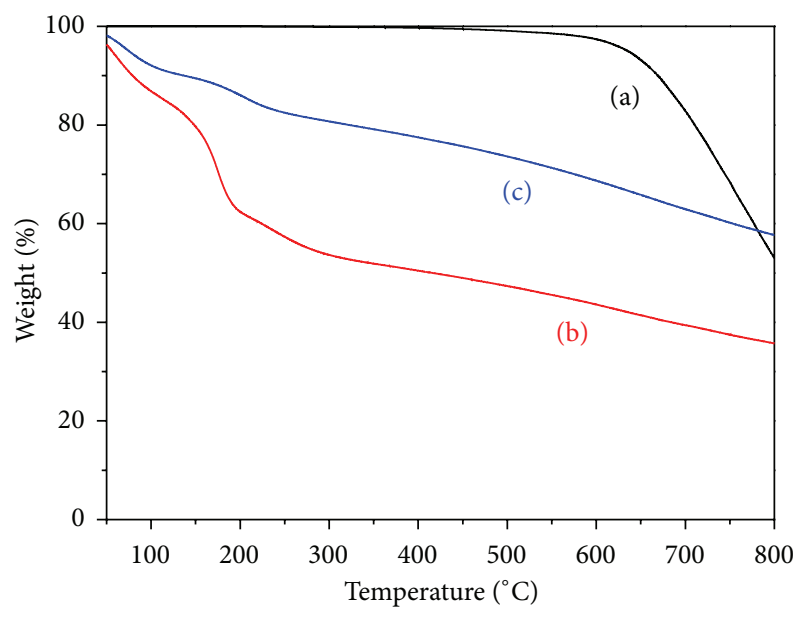

Figure 2: TGA of (a) graphite, (b) XGO, and (c) RGO.

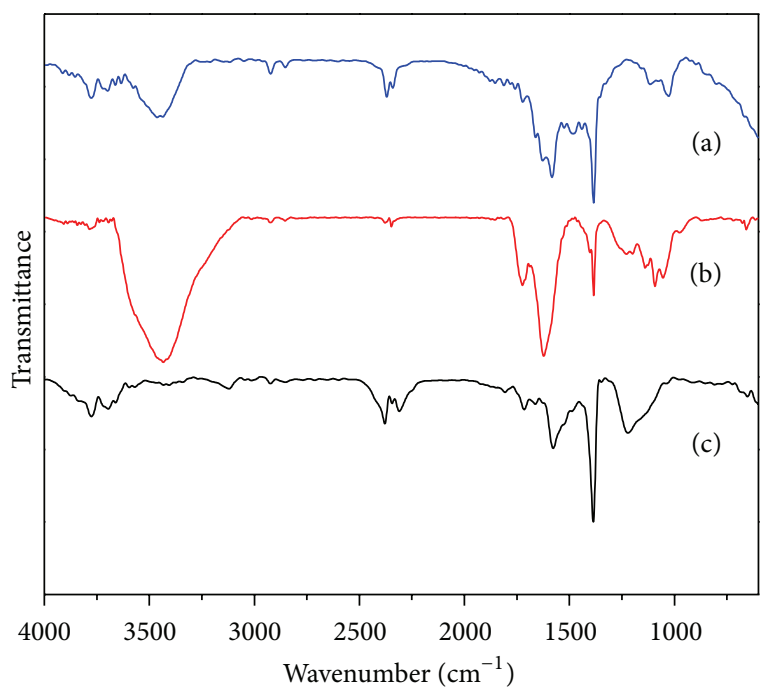

FIGURE 3: FTIR spectra of (a) graphite, (b) XGO, and (c) RGO.

of oxygen functional groups after the oxidation process. The peak at $1627 \mathrm{~cm}^{-1}$ is related to the vibrations of adsorbed water molecules. After the XGO was reduced, hydroxyl and alkoxy groups were significantly decreased (Figure 3(c)), and the phenol $\mathrm{C}=\mathrm{C}$ ring stretching at $1585 \mathrm{~cm}^{-1}$ was present [10]. In order to effectively eliminate oxygen functional groups on carbon planes, however, strongly acidic environment might be required [5].

The XRD patterns of graphite, XGO, and RGO are shown in Figure 4. Pristine graphite has a strong and sharp diffraction peak at $2 \theta=26.38^{\circ}$, corresponding to the highly organized layer structure with an interlayer distance of $0.34 \mathrm{~nm}$ along the (002) orientation [12]. After the chemical oxidation and exfoliation into XGO, however, the (002) peak is shift to lower angle at $2 \theta=10.80^{\circ}$, indicating an increase in d-spacing from $0.34 \mathrm{~nm}$ to $0.82 \mathrm{~nm}$. An increased interlayer distance between consecutive carbon basal planes is attributed to the intercalation of oxygen functional groups and water molecules into carbon layer structure $[10,13]$. The RGO,

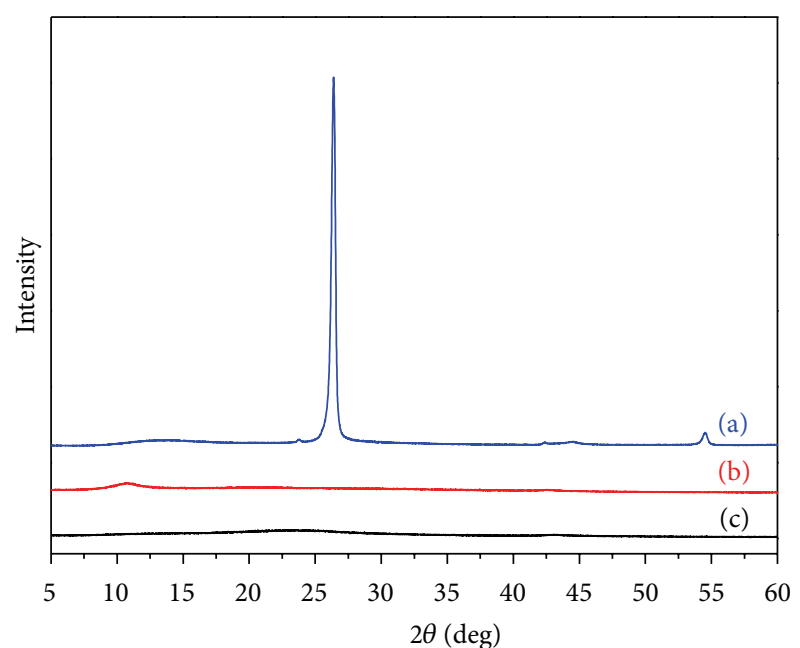

FIGURE 4: XRD patterns of (a) graphite, (b) XGO, and (c) RGO.

on the other hand, exhibits a new broad peak at $2 \theta=23.50^{\circ}$, corresponding to the d-spacing of $0.38 \mathrm{~nm}$ along the (002) orientation. This can be explained by the removal of oxygen functional groups, causing a decrease in d-spacing [14].

The UV-Visible and FT-Raman spectra of XGO and RGO are shown in Figure 5. According to the absorbance spectra, the main spectrum of XGO has an absorption peak at $230 \mathrm{~nm}$, attributed to $\pi-\pi^{*}$ transition of the aromatic $\mathrm{C}-\mathrm{C}$ ring and weak absorption at $303 \mathrm{~nm}$ due to $n-\pi^{*}$ transition of $\mathrm{C}=\mathrm{O}$ bond $[10,12]$. The peak of RGO is, however, shifted to $255 \mathrm{~nm}$ due to a decrease in oxygen functional groups and an increase in aromatic rings, causing electrons to be easily excited at a lower energy [14]. The Raman spectra of XGO and RGO reveal two prominent peaks at $\mathrm{G}$ band and $\mathrm{D}$ band. It is clearly noticed that these two bands are broader in XGO than those in RGO, corresponding to higher disorder in XGO. The sharpening of the $G$ band and an increase in the peak intensity in RGO are due to the restoring $\mathrm{sp}^{2}$ domains and the reestablished $\mathrm{sp}^{2}$ network after the reduction treatment in hot water $[15,16]$. After baseline correction, the $I_{\mathrm{D}} / I_{\mathrm{G}}$ intensity ratio of RGO is calculated to be 1.12 , which is slightly higher than that of XGO. This suggests a reduction in the average size of $\mathrm{sp}^{2}$ domains. Nevertheless, this number is much lower than those calculated from the reduction by hydrazine in previous works $\left(I_{\mathrm{D}} / I_{\mathrm{G}} \sim 1.44-1.63[17,18]\right)$. Since the $\mathrm{D}$ band is generally known as the disorder or defect band, the results illustrate that the current approach can produce XGO with less defect nature.

The morphology of the samples was studied using SEM and TEM. As shown in Figure 6(a), graphite particles are in the platelet-like crystalline form of carbon. After acid oxidation and ultrasonication, XGO sheets become smaller and transparent (Figure 6(b)). The sheet is so thin that electron beam can be passed through sample. Figures 6(c) and 6(d) show the SEM and TEM images of RGO. The reduced graphene oxide exhibits typical wrinkled structure that caused sheet folding $[19,20]$. 

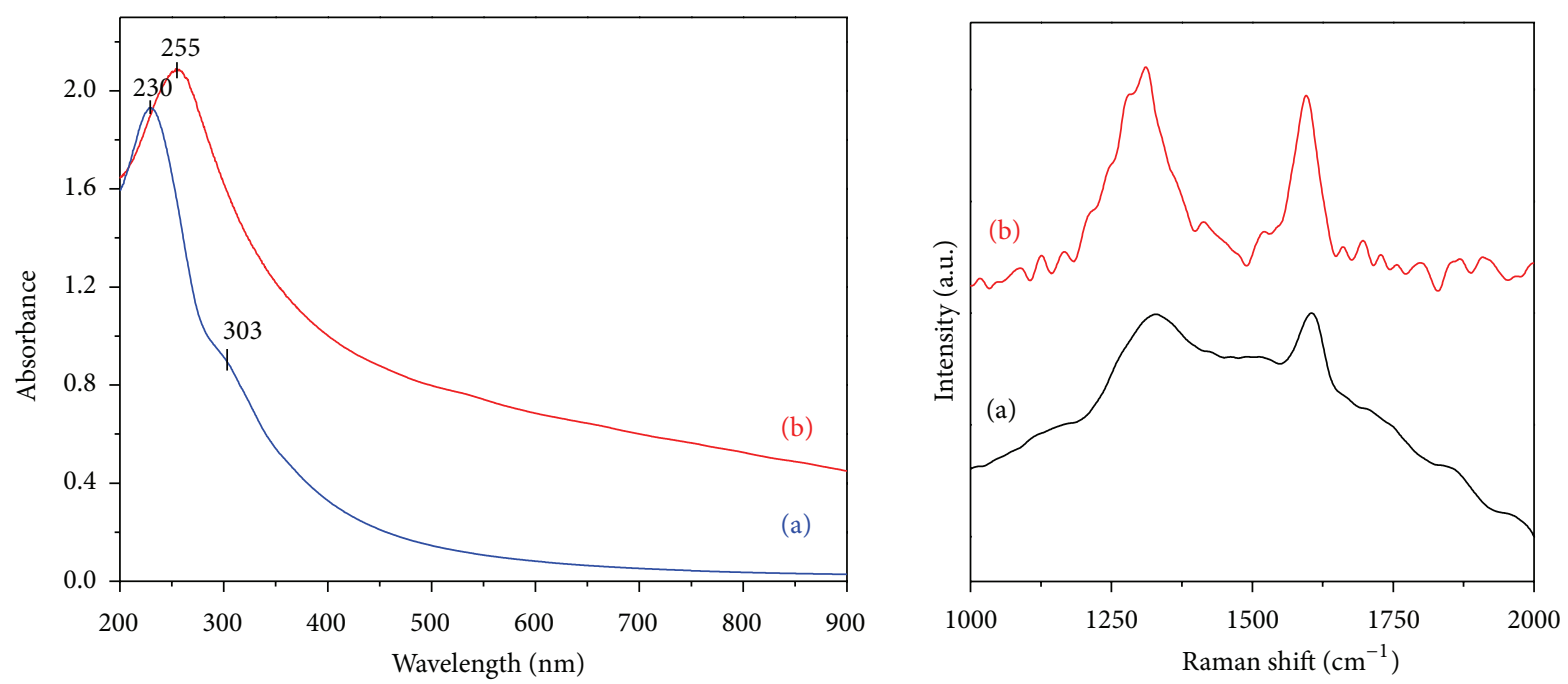

FIGURE 5: UV-Vis spectrum and FT-Raman spectra of (a) XGO and (b) RGO.
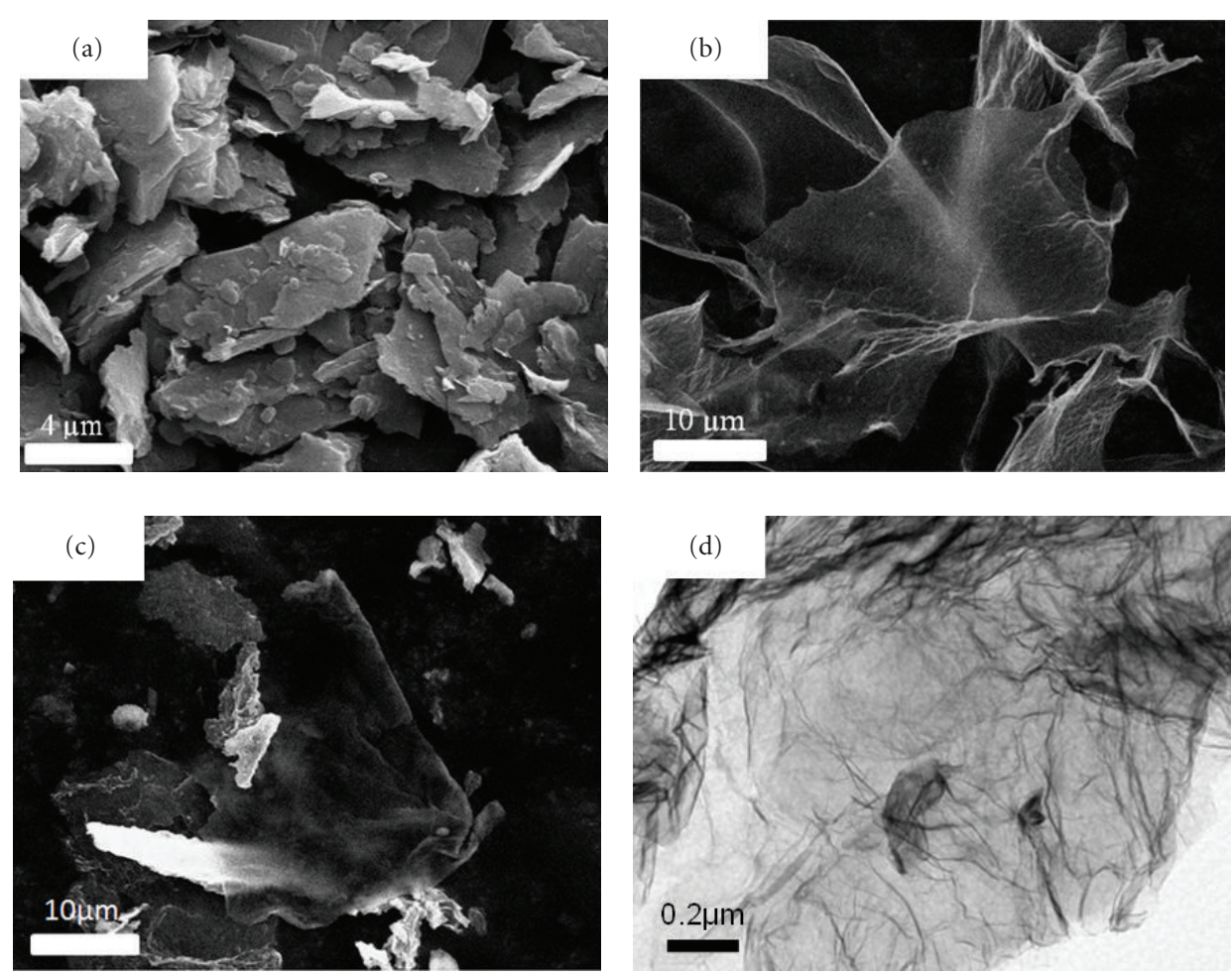

Figure 6: SEM (a, b, c) and TEM (d) images of graphite (a), XGO (b), and RGO (c, d).

\section{Conclusions}

In this study, reduced graphene oxide (RGO) can be synthesized by modified Hummer's method and reduction method by stirring in hot water. The results show that oxygen functional groups in XGO could be eliminated through stirring in hot water, as confirmed by FTIR and TGA analysis. SEM and TEM show the structures of XGO and RGO, which are very thin sheets even through there is evidence of sheet folding.

\section{Conflict of Interests}

The authors declare that there is no conflict of interests.

\section{Acknowledgments}

The authors wish to thank Department of Materials Science and Engineering, Faculty of Engineering and Industrial 
Technology, Silpakorn University, and National Center of Excellence for Petroleum, Petrochemicals and Advanced Materials for supporting and encouraging this investigation.

\section{References}

[1] A. A. Arbuzov, V. E. Muradyan, and B. P. Tarasov, "Synthesis of few-layer graphene sheets via chemical and thermal reduction of graphite oxide," in Proceedings of the International Conference Nanomaterials: Applications and Properties, 2012.

[2] V. Singh, D. Joung, L. Zhai, S. Das, S. I. Khondaker, and S. Seal, "Graphene based materials: past, present and future," Progress in Materials Science, vol. 56, no. 8, pp. 1178-1271, 2011.

[3] K. Wang, T. Feng, M. Qian, H. Ding, Y. Chen, and Z. Sun, “The field emission of vacuum filtered graphene films reduced by microwave," Applied Surface Science, vol. 257, no. 13, pp. 58085812, 2011.

[4] S. Pei and H. M. Cheng, "The reduction of graphene oxide," Carbon, vol. 50, no. 9, pp. 3210-3228, 2012.

[5] K.-H. Liao, A. Mittal, S. Bose, C. Leighton, K. A. Mkhoyan, and C. W. MacOsko, "Aqueous only route toward graphene from graphite oxide," ACS Nano, vol. 5, no. 2, pp. 1253-1258, 2011.

[6] J. Wu, X. Shen, L. Jiang, K. Wang, and K. Chen, "Solvothermal synthesis and characterization of sandwich-like graphene/ZnO nanocomposites," Applied Surface Science, vol. 256, no. 9, pp. 2826-2830, 2010.

[7] S. Park and R. S. Ruoff, "Chemical methods for the production of graphenes," Nature Nanotechnology, vol. 5, no. 4, pp. 217-224, 2009.

[8] S. Stankovich, D. A. Dikin, R. D. Piner et al., "Synthesis of graphene-based nanosheets via chemical reduction of exfoliated graphite oxide," Carbon, vol. 45, no. 7, pp. 1558-1565, 2007.

[9] Z. Fan, K. Wang, T. Wei, J. Yan, L. Song, and B. Shao, "An environmentally friendly and efficient route for the reduction of graphene oxide by aluminum powder," Carbon, vol. 48, no. 5, pp. 1686-1689, 2010.

[10] M. El Achaby, F. Z. Arrakhiz, S. Vaudreuil, E. M. Essassi, and A. Qaiss, "Piezoelectric $\beta$-polymorph formation and properties enhancement in graphene oxide-PVDF nanocomposite films," Applied Surface Science, vol. 258, no. 19, pp. 7668-7677, 2012.

[11] A. Bagri, C. Mattevi, M. Acik, Y. J. Chabal, M. Chhowalla, and V. B. Shenoy, "Structural evolution during the reduction of chemically derived graphene oxide," Nature Chemistry, vol. 2, no. 7, pp. 581-587, 2010.

[12] S. Thakur and N. Karak, "Green reduction of graphene oxide by aqueous phytoextracts," Carbon, vol. 50, no. 14, pp. 5331-5339, 2012.

[13] M. Wojtoniszak, X. Chen, R. J. Kalenczuk et al., "Synthesis, dispersion, and cytocompatibility of graphene oxide and reduced graphene oxide," Colloids and Surfaces B, vol. 89, pp. 79-85, 2012.

[14] F. T. Thema, M. J. Moloto, E. D. Dikio et al., "Synthesis and characterization of graphene thin films by chemical reduction of exfoliated and intercalated graphite oxide," Journal of Chemistry, vol. 2013, Article ID 150536, 6 pages, 2013.

[15] K. N. Kudin, B. Ozbas, H. C. Schniepp, R. K. Prud'homme, I. A. Aksay, and R. Car, "Raman spectra of graphite oxide and functionalized graphene sheets," Nano Letters, vol. 8, no. 1, pp. 36-41, 2008.

[16] H. Yang, W. Zhou, B. Yu, Y. Wang, C. Cong, and T. Yu, "Uniform decoration of reduced graphene oxide sheets with gold nanoparticles," Journal of Nanotechnology, vol. 2012, Article ID 328565, 8 pages, 2012.

[17] V. C. Tung, M. J. Allen, Y. Yang, and R. B. Kaner, "Highthroughput solution processing of large-scale graphene," Nature Nanotechnology, vol. 4, no. 1, pp. 25-29, 2009.

[18] Y. Zhou, Q. Bao, L. A. L. Tang, Y. Zhong, and K. P. Loh, "Hydrothermal dehydration for the "green" reduction of exfoliated graphene oxide to graphene and demonstration of tunable optical limiting properties," Chemistry of Materials, vol. 21, no. 13, pp. 2950-2956, 2009.

[19] H. Zhang and P. X. Feng, "Fabrication and characterization of few-layer graphene," Carbon, vol. 48, no. 2, pp. 359-364, 2010.

[20] L. Ortolani, E. Cadelano, G. P. Veronese et al., "Folded graphene membranes: mapping curvature at the nanoscale," Nano Letters, vol. 12 , no. 10 , pp. $5207-5212$. 

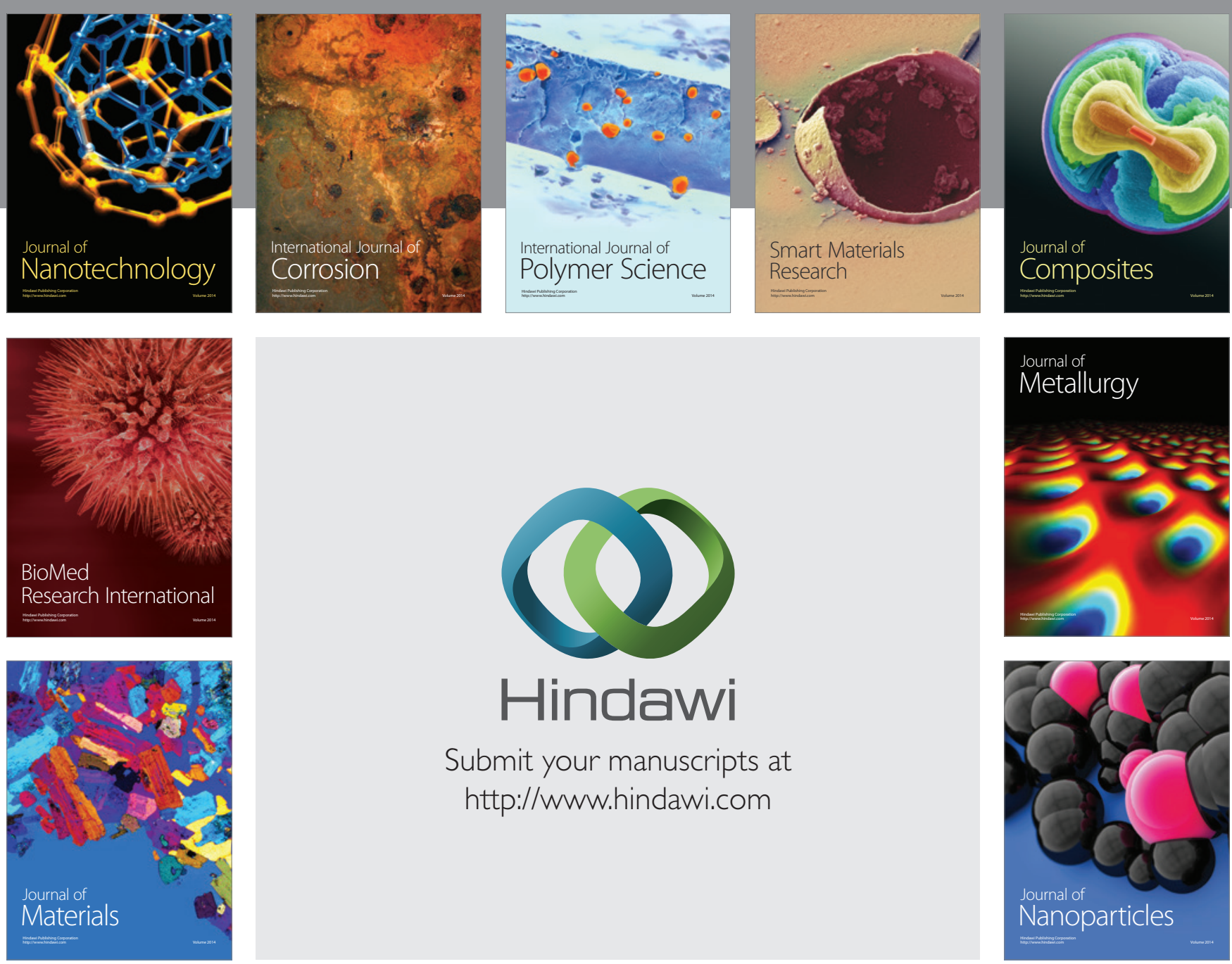

Submit your manuscripts at http://www.hindawi.com
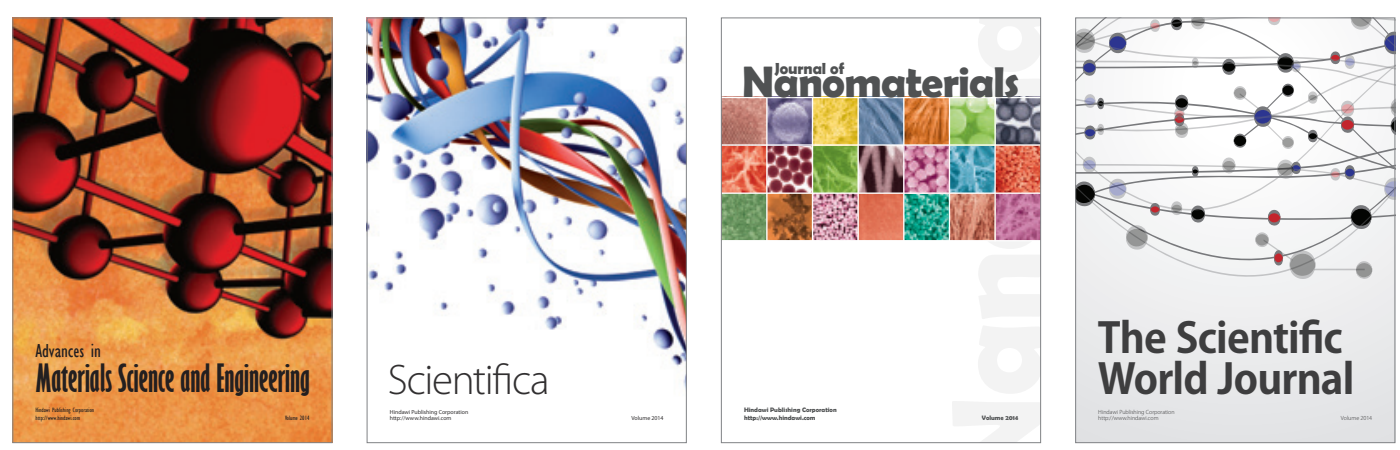

\section{The Scientific World Journal}
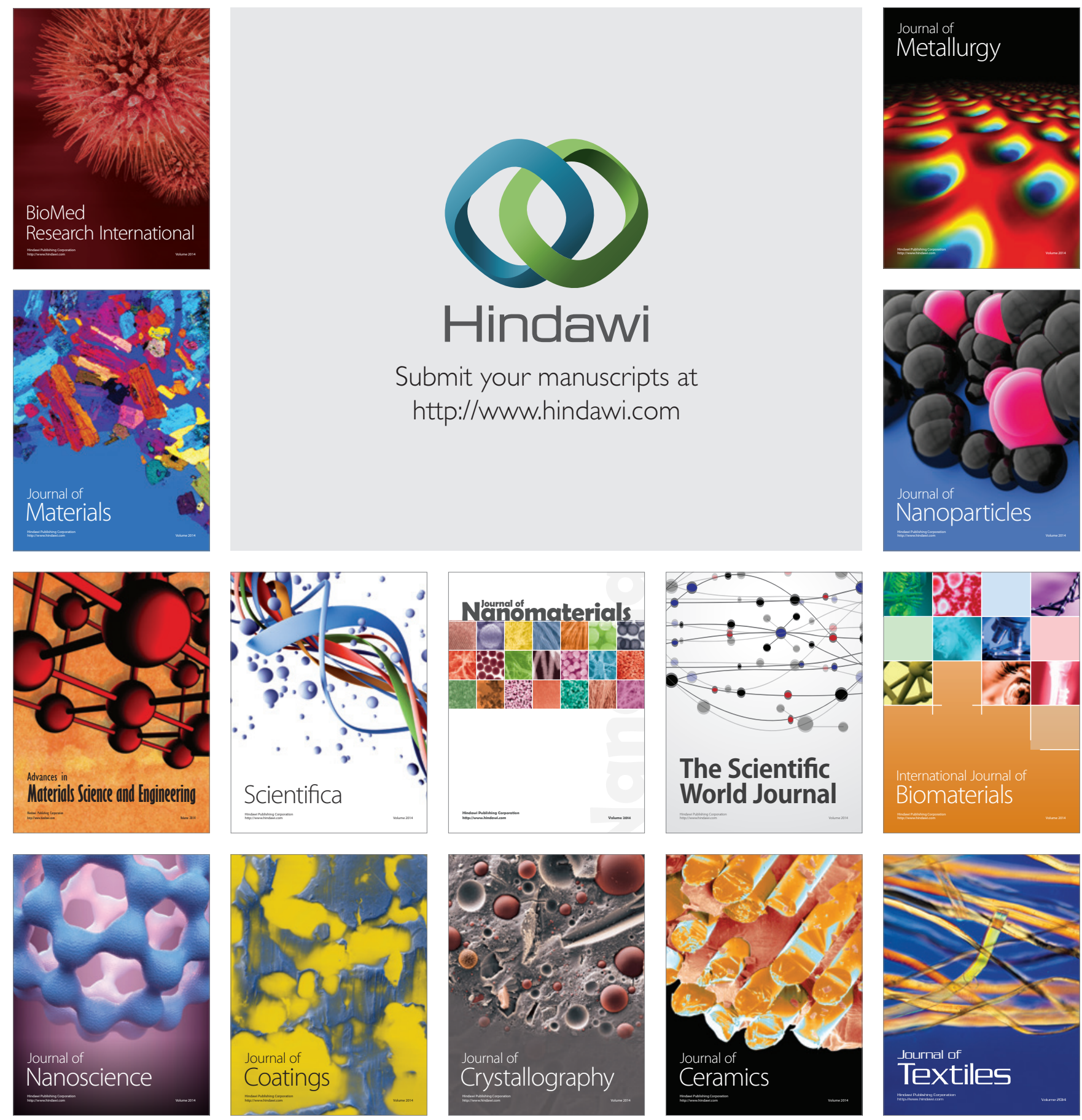